\title{
A fitness index for transplantation of machine-perfused cadaveric rat livers
}

Sinem Perk ${ }^{1 \dagger}$, Maria-Louisa Izamis ${ }^{1 \dagger}$, Herman Tolboom² , Basak Uygun ${ }^{1}$, Martin L Yarmush ${ }^{1,3}$ and Korkut Uygun ${ }^{1 *}$

\begin{abstract}
Background: The 110,000 patients currently on the transplant waiting list reflect the critical shortage of viable donor organs. However, a large pool of unused organs, from donors after cardiac death (DCD) that are disqualified because of extensive ischemic injury, may prove transplantable after machine perfusion treatment, fundamentally impacting the availability of treatment for end-stage organ failure. Machine perfusion is an ex-vivo organ preservation and treatment procedure that has the capacity to quantitatively evaluate and resuscitate cadaveric organs for transplantation.

Methods: To diagnose whether an organ was fresh or ischemic, an initial assessment of liver quality was conducted via dynamic discriminant analysis. Subsequently, to determine whether the organs were sufficiently viable for successful implantation, fitness indices for transplantation were calculated based on squared prediction errors (SPE) for fresh and ischemic livers.

Results: With just three perfusate metabolites, glucose, urea and lactate, the developed MPLSDA model distinguished livers as fresh or ischemic with 90\% specificity. The SPE analyses revealed that fresh livers with $\mathrm{SPE}_{\mathrm{F}}<10.03$ and WI livers with SPE $\mathrm{WI}_{1}<3.92$ yield successful transplantation with 95\% specificity.

Conclusions: The statistical methods used here can discriminate between fresh and ischemic livers based on simple metabolic indicators measured during perfusion. The result is a predictive fitness index for transplantation of rat livers procured after cardiac death. The translational implications of this study are that any donor organ procured from controlled, but most especially from uncontrolled cardiac death donors, will be objectively assessed and its recovery monitored over time, minimizing the critical loss of otherwise viable organs.
\end{abstract}

Keywords: Transplantation index, Principal component analysis (PCA), Partial least squares (PLS), Extracorporeal liver perfusion, Donors after cardiac death

\section{Background}

The major untapped pool of donor organs that could be used to alleviate the critical shortage of transplantations derives from donors who have experienced cardiac death $[1,2]$. Approximately 4,000 patients perish while waiting for a donor liver every year [3], while the estimated pool of livers from donors after cardiac death (DCD) with recoverable ischemic times of $30-60$ minutes is on the order of 6,000 grafts per year [2].

\footnotetext{
* Correspondence: uygun.korkut@mgh.harvard.edu

${ }^{\dagger}$ Equal contributors

${ }^{1}$ Center for Engineering in Medicine, Massachusetts General Hospital, Harvard Medical School, and the Shriners Hospitals for Children, 51 Blossom Street, Boston, MA 02114, USA

Full list of author information is available at the end of the article
}

In the absence of cardiac output, ischemic damage increases in severity as a function of time. Beyond a certain cutoff (about 30 minutes for the liver) graft survival in the recipient falls dramatically [4]. Preclinical studies with extracorporeal machine perfusion systems in porcine and murine models of DCD livers [5-11], including from our group [12-14], indicate that up to 60 minutes of warm ischemic damage can be successfully reversed, whereas static cold storage in preservation medium, the current clinical gold standard, simply exacerbates the damage and recipient animals do not survive. Research in machine perfusion systems is subsequently a very active field in donor organ recovery and preservation [15-18].

In humans, cardiac death frequently occurs in uncontrolled environments $(\mathrm{uDCD})$. Hence without objective

\section{Biomed Central}


metrics of ischemic duration and organ viability, uDCD organs cannot be safely transplanted. A suitable framework for developing a fitness index that describes whether an organ is transplantable or not could be based on the dynamic correlation between metabolites measured during perfusion and evaluated with multivariate statistical process monitoring (SPM) [19,20]. Normothermic $\left(37{ }^{\circ} \mathrm{C}\right)$ Machine Perfusion (NMP) is especially amenable to this analysis since it allows measurable metabolic activity to occur in the organ.

The aim of this work is the development of a fitness index for transplantation for DCD livers based on the organ ischemic injury level and metabolite transient profiles during perfusion, as a proof-of-concept in a rat model. To create such an index, we compared NMP treatment of fresh, $60 \mathrm{~min}$, and $90 \mathrm{~min}$ warm ischemic (WI) livers, where only the last group did not survive transplantation. We first constructed a multi-way partial least squares discriminant analysis (MPLSDA) model to assess what the acceptable degree of ischemic damage of each liver was for successful transplantation $(>1$ month survival) and classified them as fresh or ischemic based on their urea, lactate and glucose profiles during perfusion. We also constructed fresh liver and ischemic liver successful perfusion models using multi-way principal component analysis (MPCA). Once a new liver was identified as fresh or ischemic, the corresponding MPCA model determined the SPE-distance of the given liver to the reference livers used in model-building, hence, constructing a predictive fitness index for transplantation based on SPE statistics. If the fitness index for the new liver fell within the $99 \%$ confidence limits, this would indicate that the liver is suitable for transplantation.

\section{Methods}

Animals

Male Lewis Rats weighing 200-300 g were obtained from Charles River Laboratories (Wilmington, MA) and maintained in accordance with National Research Council guidelines. The Subcommittee on Research Animal Care, Committee on Research at the Massachusetts General Hospital, approved the experimental protocols. All animals were allowed to acclimatize for at least 2 days prior to any experimentation. Full details of the liver isolation protocol for donor livers are explained in [14]. WI was induced by placing livers in a temperature-controlled chamber filled with saline and maintained at $34 \pm 0.1{ }^{\circ} \mathrm{C}$ during which time the portal vein and vena cava were cuffed. Ex vivo ischemia ensured a constant temperature [21] and enabled a severe model of warm ischemia [8]. Livers that have been exposed to 60 minute or 90 minute warm ischemia were flushed with saline and then connected to the perfusion system. Fresh livers were flushed through the PV with $10 \mathrm{mls}$ of saline upon clamping the vein in situ and were then placed in a bowl of room temperature saline to be cuffed at the PV and IVC; average warm ischemic time prior to reperfusion was $10 \pm 2$ minutes. Procured livers were grouped in three with respect to the duration of warm ischemia they were exposed to: 1) Fresh (F; $\mathrm{n}=10), 2) 60 \mathrm{~min}$ warm ischemia (WI; $n=6$ ), and 3) 90 min warm ischemia (WI90; $\mathrm{n}=3)$.

\section{Normothermic liver perfusion and transplantation}

The perfusion medium contained phenol red-free Williams Medium E (WE, Sigma Chemical, St. Louis, MO). WE was supplemented with $2 \mathrm{u} / \mathrm{L}$ insulin $(28.85$ units/mg Humulin, Eli Lily, Indianapolis, IN), 100,000 u/L penicillin, $100 \mathrm{mg} / \mathrm{L}$ streptomycin sulfate (Gibco, Invitrogen, Grand Island, NY), 0.292 g/L L-glutamine (Gibco), 10 mg/L hydrocortisone (Solu-Cortef, Pharmacia \& Upjohn, Kalamazoo, MI), and $1000 \mathrm{u} / \mathrm{L}$ heparin (APP, Schaumburg, IL). The primary circuit of the perfusion system comprised perfusion medium (perfusate) that recirculated by means of a peristaltic pump through a jacketed perfusion chamber, a membrane oxygenator, a heat exchanger, and a bubble trap. The oxygenator was gassed with a mixture of $74 \% \mathrm{~N}_{2} / 21 \% \mathrm{O}_{2} / 5 \% \mathrm{CO}_{2}$ and $100 \% \mathrm{O}_{2}$ to maintain a constant $\mathrm{pH}$. Fresh rat plasma $(25 \% \mathrm{v} / \mathrm{v})$ and erythrocytes $(18-20 \% \mathrm{v} / \mathrm{v})$ were collected earlier and added to the perfusate. The total perfusate volume was 55 to $60 \mathrm{~mL}$. Perfusate hematocrit was sustained, nutrients were replenished, and metabolism by-products were diluted through dialysis. A hollow fiber dialyzer with a $2200 \mathrm{~cm}^{2}$ membrane area and a $30 \mathrm{kDa}$ nominal molecular cutoff weight (SpectrumLabs, Rancho Dominguez, CA) enabled counter-current mixing of perfusate in the primary circuit with a reservoir of WE (dialysate) in a secondary circuit. Temperature within the system was maintained at $37^{\circ} \mathrm{C}$.

Upon completion of cuffing of fresh livers ( $5 \mathrm{~min})$ and after the period of warm ischemia for WI livers, they were immersed in perfusate in the perfusion chamber. Livers were perfused at a constant flow rate through the portal vein while maintaining portal pressure between 10 and $12 \mathrm{cmH}_{2} \mathrm{O}$. The effluent flowed freely from the SHVC and IVC into the surrounding medium. When the recipient hepatectomy was prepared, the liver was disconnected from the circuit, rinsed in a bowl of saline at room temperature, and weighed again before transplantation. A modification of the cuff technique designed by Kamada and Calne [22-24] was used for orthotopic rat liver transplantation. The anhepatic phase of the procedure was typically 13-15 minutes and did not exceed 17 minutes. Animals were hydrated with $8 \mathrm{ml} / \mathrm{kg}$ of warm $\left(37^{\circ} \mathrm{C}\right)$ lactated Ringer's solution with $5 \%$ dextrose and $2 \mathrm{ml} / \mathrm{kg}$ of $\mathrm{NaHCO}_{3} 7 \% \mathrm{w} / \mathrm{v}$ (Abbott, North Chicago, IL) by penile vein injection. Following 
transplantation, the animals were allowed to recover from anesthesia in separate cages under an infrared lamp for half an hour, and subsequently returned to regular housing. During the first 12 hours post-transplantation animals were checked every 2 hours and subsequently every 8 hours for one week, and daily afterwards.

\section{Metabolite sampling}

Perfusate and dialysate samples $(1 \mathrm{~mL})$ were collected hourly from the liver effluent and reservoir, respectively. Perfusate samples were first spun down at $3000 \mathrm{~g}$ before storing the supernatant at $-80^{\circ} \mathrm{C}$. We focused on readilymeasured metabolites: Urea was assayed by reaction with diacetyl monoxime using a commercial assay kit (BUN, Sigma-Aldrich, St. Louis, MO). Lactate was measured using the enzymatic conversion to pyruvate and hydrogen peroxide with lactate oxidase from a commercially available kit (Trinity Biotech, Berkeley Heights, NJ). Glucose measurements were quantified with an enzymatic assay kit through conversion to 6-phospogluconate (Glucose assay kit, Sigma). Data consisted of glucose, lactate, and urea measured hourly for each perfusion. 10 fresh livers, 6 (60 $\mathrm{min})$ and 3 (90 $\mathrm{min})$ WI livers were each perfused for 6 hours. Fresh livers and livers that were exposed to 60 minutes of WI were successfully transplanted with $>1$ month survival (Table 1 ).

\section{Statistical analysis}

Statistical process control methodologies for batch processes were used in the analyses. Each perfusion is a batch process with a finite duration. The process data is a 3D array that consists of (perfusions $x$ number of variables $\mathrm{x}$ time). An ( $\mathrm{x} J \mathrm{x} \mathrm{K}$ ) data array can be unfolded by preserving the batch direction I and augmenting the $\mathrm{J}$ variable measurements taken at each time point $k$ $(\mathrm{k}=1, \ldots, \mathrm{K})$ side by side resulting in an $\mathrm{I} \mathrm{x} \mathrm{JK}$ matrix $[19,20,25,26]$. This unfolding direction is the best to use in the SPM of batch processes since it considers the batch-to-batch variations. The (I x JK) unfolding of the batch data yields datasets which consist of few observations and many variables. Unlike ANOVA and classical methods of statistics, the multivariate projection methods such as MPCA, MPLSDA work effectively on these short and wide datasets with constrained sample sizes [19]. 10 fresh liver perfusions and 6 WI liver perfusions were statistically sufficient for modeling using SPM methodologies. An additional three replicates were performed with 90 minute WI liver perfusions for testing. Please note that unlike ANOVA designs, equal replicates in all groups is not necessary. All variables (i.e. the metabolite concentrations) were mean-centered and unit-variance scaled for further statistical analyses.

\section{Multiway partial least square discriminant analysis (MPLSDA)}

All Fresh and 60 min WI liver perfusions were transplanted successfully. However, during perfusion $60 \mathrm{~min}$ warm ischemic livers displayed significantly different metabolic profiles compared to fresh livers hence, indicating the need for the fitness for transplantation to be evaluated separately for reversibly ischemic and fresh livers. To achieve this, first the quality of the liver had to be determined as fresh or $60 \mathrm{~min}$ WI.

To be able to classify livers as ischemic or fresh, an algorithm that can discriminate different metabolic states from the dynamic metabolite data collected during successful perfusions is required. Partial least squares or projections to latent structures (PLS) is a regression technique that is used to connect the information in two blocks of variables, namely the predictor block $\mathbf{X}$ and response block Y. An algorithm for Nonlinear Iterative Partial Least Squares (NIPALS) is provided in [27]. PLS is extended to multi-way PLS (MPLS) for three dimensional (3D) data as (perfusions $\mathrm{x}$ variables $\mathrm{x}$ time). For new data coming from a new perfusion, $\mathbf{x}_{\text {new }}$, MPLS computes the response variables $\mathbf{y}_{\text {new }}$ using the model regression coefficients.

MPLS discriminant analysis (MPLSDA) is used to classify observations or independent experiments in $\mathbf{X}$ as belonging to one of several a-priori known classes in $\mathbf{Y}$ based on their relationship [19]. The predictive power of MPLSDA was evaluated by case-re-sampling cross validation technique $[28,29]$. In this approach, the data set was sampled randomly multiple times to create training and validation data sets. For each sampling, five samples from the fresh liver group and five samples from the $60 \mathrm{~min}$ WI liver group were selected randomly and the remaining samples were used for testing the model. The maximum selection threshold, which is the maximum number of times same perfusion can be used in the same model, was set to 2 . The selection threshold was added to avoid the same samples being selected most of the time.

For the analysis of fresh and ischemic livers, $\mathbf{X}$ consists of the three metabolites' six hour trajectories for ten

Table 1 Initial conditions and number of grafts for 6 hours of machine-perfusion

\begin{tabular}{ccc}
\hline Initial condition & Total number of livers used in analysis & Transplant result \\
\hline Fresh Livers & 10 & Survival (10/10) \\
60 min warm ischemia (WI) & 6 & Survival (6/6) \\
90 min WI & 3 & Failure (3/3) \\
\hline
\end{tabular}


selected perfusions: five 60 minute WI and five fresh; and Y consists of two columns representing the class membership of each of the ten batches. For a fresh liver perfusion batch (class 1), the corresponding row of $\mathbf{Y}$ is $[1,0]$, whereas, for a WI liver batch the row is set to $[0,1]$. When the new data $\mathbf{x}_{\text {new }}$ is projected onto the MPLSDA model, $y$ vector is calculated. The predicted class is the numerical maximum of the normalized vector.

\section{Multi-way principal component analysis (MPCA)}

For the statistical modeling of liver perfusions with MPCA, two MPCA models were built to capture the metabolic functioning of fresh and warm ischemic (60 minute) livers during perfusions.

PCA captures the correlation structure between the variables in $\mathbf{X}$ and forms a model plane with fewer dimensions ( $\mathrm{R}$ principal component (PC) directions) using only the $R$ largest variance directions [30]. $R$ is chosen such that adding additional components to the model does not provide additional significant information. Instead of working with highly correlated collinear variables $(\mathbf{X})$, PCA yields fewer and uncorrelated projections (scores $(\mathbf{T})$ ).

$$
\begin{aligned}
\mathbf{X} & =\mathbf{T} \mathbf{P}^{\mathbf{T}}+\mathbf{E}=\mathbf{X}+\mathbf{E} ; \quad \hat{\mathbf{t}}=\mathbf{x}_{\text {new }} \mathbf{P} ; \quad \mathbf{e}_{\text {new }} \\
& =\mathbf{x}_{\text {new }}-\mathbf{t} \mathbf{P}^{T}
\end{aligned}
$$

PCA is performed by singular value decomposition in the covariance of $\mathbf{X}$ and the loadings (eigenvectors) $\mathbf{P}$ are derived. The $\mathrm{R}$ eigenvectors are the $\mathrm{R}$ highest variance directions. Scores $(\mathbf{T})$ are the new uncorrelated variable projections onto the newly derived PCA plane. $\mathbf{E}$ is the residual matrix. Score bi-plots can then be used to reveal the degree of similarity between independent samples, possible clustering among the samples, and any outliers. Similar to MPLS, PCA has been extended to MPCA for 3D arrays $[20,25,31]$ to handle the time-series data obtained during perfusion.

\section{Squared prediction error (SPE)}

SPE statistic $[20,25,26,31,32]$ captures the large deviations from a reference set and is calculated for the new batch using the residuals $\mathbf{e}_{\text {new }}$ [20],

$$
S P E=\mathbf{e}_{n e w} \mathbf{e}_{\text {new }}^{T}=\sum_{c=1}^{K J} E_{\text {new }, c}^{2}
$$

A large SPE statistic indicates that the observation under consideration contains a different correlation structure and is not explained by the model. $X_{2 m^{2} / v, \alpha}^{2}$ is the critical value of the chi-squared variable with $2 \mathrm{~m}^{2} / \mathrm{v}$ degrees of freedom at significance level $\alpha . m$ and $v$ are the sample mean $(\mathrm{m})$ and variance $(\mathrm{v})$ of the SPE values of the reference batches [25].

For instance, if a given liver perfusion contains a correlation structure that is not captured by the MPCA model, it will be indicated by a large SPE value. As such, SPE effectively distills the dynamic metabolite profiles to a single variable that quantifies the distance of any liver perfusion from a set of reference perfusions. For fresh livers, MPCA model 99\% confidence limits will determine the maximum acceptable value of the SPE statistic

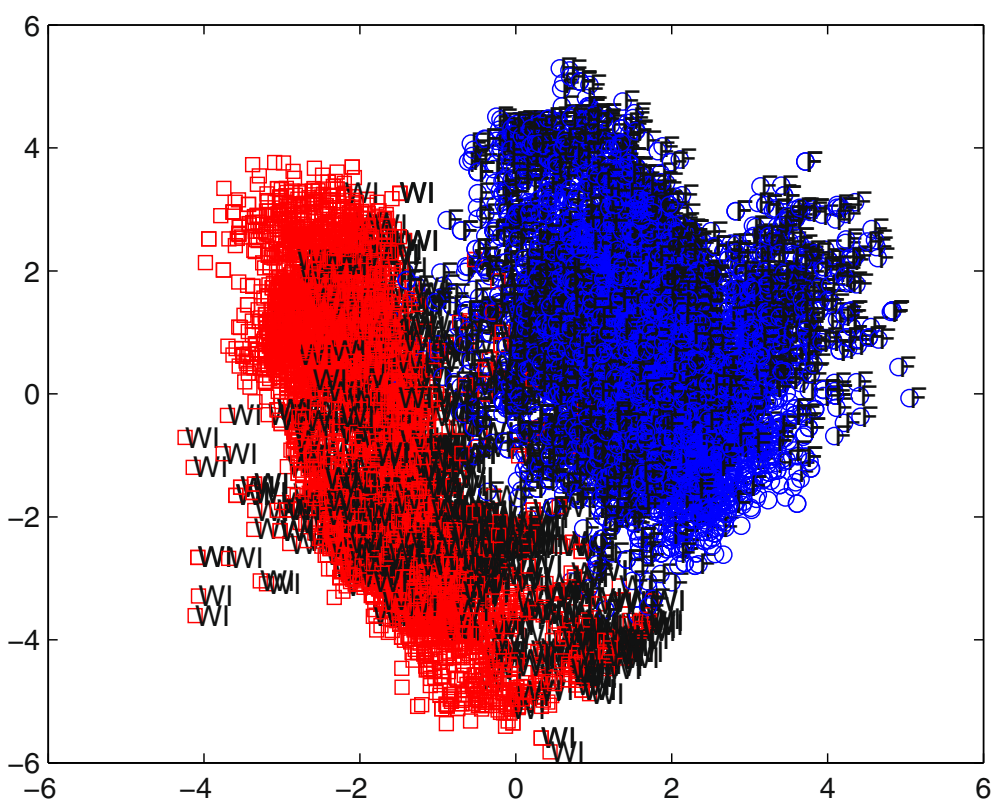

Figure 1 MPLSDA fresh and 60 minute WI liver clusters. Red squares and blue circles denote ischemic livers and fresh livers, respectively. 


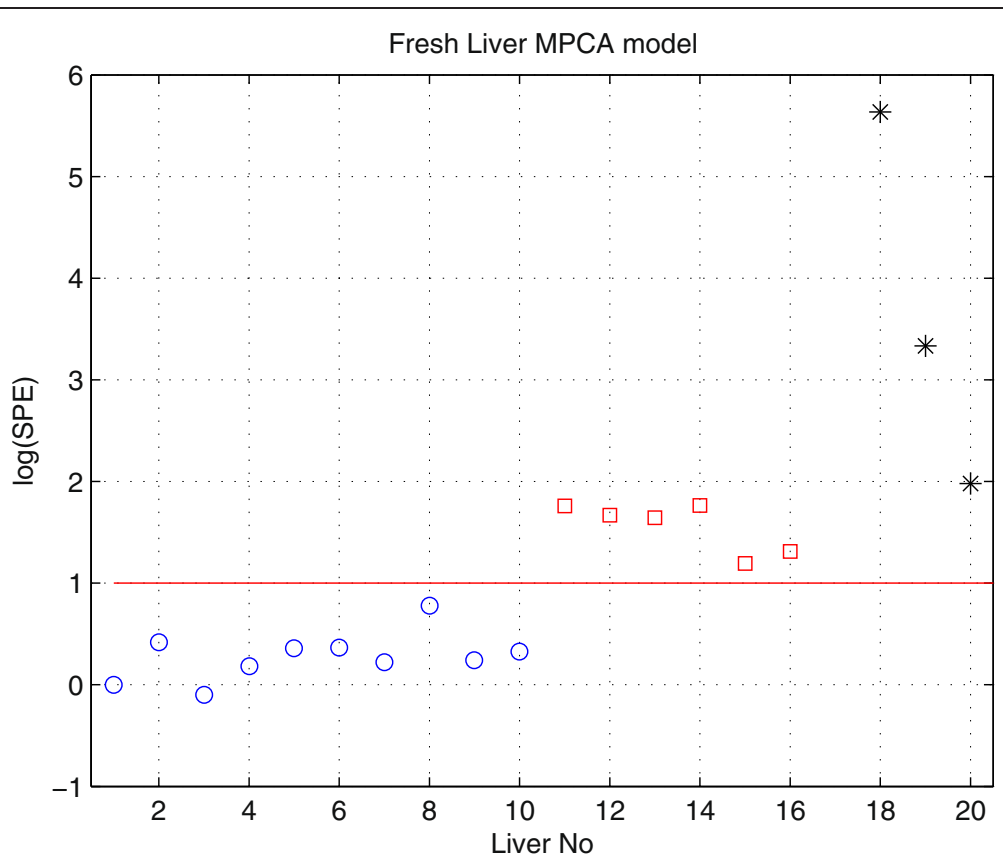

Figure 2 Log(SPE) distances to the fresh liver MPCA model. Fresh livers, denoted by blue circles, are within the $99 \%$ model confidence limits $\left(\mathrm{SPE}_{\mathrm{F}}=10.03\right) .60 \mathrm{~min}$ and $90 \mathrm{~min}$ ischemic livers are denoted by red squares and black stars, respectively.

for a fresh liver perfusion to be deemed fit for transplantation. Similarly, MPCA model of transplantable ischemic livers will determine the upper limit for the SPE statistic for an ischemic liver to be considered transplantable.

\section{Results}

\section{Degree of ischemia and classification}

MPLSDA can classify a perfused graft as healthy or ischemic so that a decision can be made about the organ quality and eventually, on its fitness for transplantation

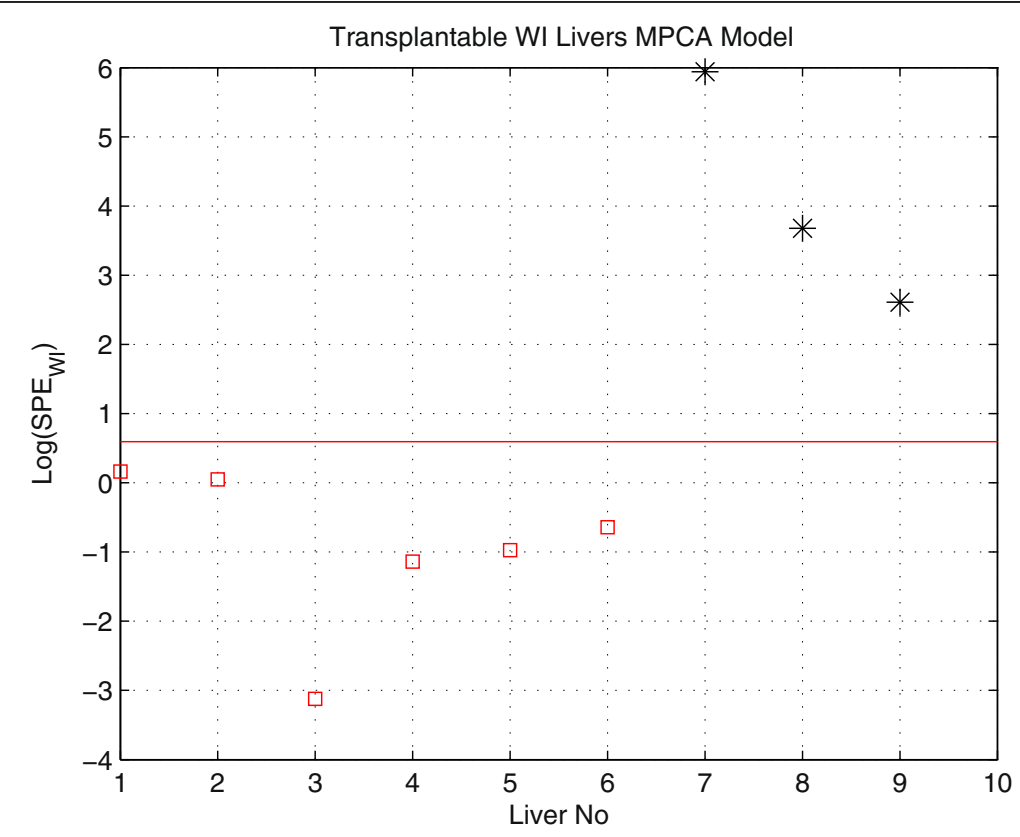

Figure 3 Log(SPE) distances to the 60 min WI liver MPCA model. 60 min WI livers, which were successfully transplanted, are denoted by red squares. The $99 \%$ model confidence limits (SPE $\mathrm{WI}=3.92$ ). 90 min ischemic livers, denoted by black stars, are outside the confidence limits. 
by comparing it to sets of successfully transplanted fresh and ischemic livers.

Five fresh liver and five $60 \mathrm{~min}$ WI liver perfusions were used in the MPLSDA model $(\mathrm{R}=4), 4$ PCs explain more than $86 \%$ of the variation in $\mathbf{X}$ and $99 \%$ variation in $\mathbf{Y}$.

Two distinct clusters were observed in the score plot (Figure 1). $60 \mathrm{~min}$ ischemic livers and fresh livers have different urea, lactate, and glucose concentration profiles; hence it was evident that the two groups should be modeled separately for the determination of separate fitness indices for transplantation.

\section{Fitness for transplantation indices for fresh and ischemic livers}

Once the MPLSDA determined whether a liver was fresh or ischemic, the corresponding MPCA model and its 99\% SPE confidence limits was used as a basis to determine the organ's fitness for transplantation. Accordingly, we considered SPE statistic as a heuristic index for rats for fitness of transplantation, with $99 \%$ confidence values of $\mathrm{SPE}_{\mathrm{F}}<10.03$ and $\mathrm{SPE}_{\mathrm{WI}}<3.92$ indicating fitness.

The transplantable fresh livers model and the transplantable ischemic livers model are shown in Figures 2 and 3. In the fresh liver MPCA model, the $60 \mathrm{~min}$ and the $90 \mathrm{~min}$ ischemic livers are outside of the limits since they do not retain the same concentration profiles of transplantable fresh livers (Figure 2). The SPE values for $90 \mathrm{~min}$ WI livers are the largest, indicating a larger qualitative difference to fresh livers than $60 \mathrm{~min}$ WI livers. Next, the 90 min WI liver perfusions were projected onto the 60 min WI liver perfusions MPCA model. The 99\% confidence limit, $\mathrm{SPE}_{\mathrm{WI}}<3.92$, for transplantable warm ischemic livers was used and all of the 90 min WI livers were outside the limits, affirming that these livers were not fit for transplantation (Figure 3).

The classification followed by SPE calculation scheme for the determination of transplantation fitness yields the results given in Table 2. For each liver, the incorrect predictions are summed and averaged over the number of times the liver has been picked for model-testing out of 1000 runs. The incorrect predictions are due to the deficiency of the MPLSDA classification (specificity $90 \%$ ), where some of the fresh livers are confused as ischemic livers. This scheme is able to successfully predict (no misclassifications) that the $90 \mathrm{~min}$ ischemic livers were not fit for transplantation.

\section{Discussions and conclusion}

In this work we introduce statistical process monitoring methodologies that employ liver metabolic performance to evaluate the degree of ischemic injury cadaveric organs have sustained and, based on the degree of injury, determine the fitness for transplantation.
Table 2 Fitness for transplantation predictions

\begin{tabular}{cccccc}
\hline $\begin{array}{c}\text { Fresh } \\
\text { liver } \\
\text { No }\end{array}$ & $\begin{array}{c}\text { Incorrect } \\
\text { predictions* } \\
\text { (\%) }\end{array}$ & $\begin{array}{c}\mathbf{6 0} \text { min } \\
\text { WI liver } \\
\text { No }\end{array}$ & $\begin{array}{c}\text { Incorrect } \\
\text { predictions* } \\
\text { (\%) }\end{array}$ & $\begin{array}{c}\mathbf{9 0} \text { min } \\
\text { WI liver } \\
\text { No }\end{array}$ & $\begin{array}{c}\text { Incorrect } \\
\text { predictions* } \\
\text { (\%) }\end{array}$ \\
\hline 1 & 10.08 & 1 & 0 & 1 & 0 \\
2 & 1.53 & 2 & 0 & 2 & 0 \\
3 & 21.35 & 3 & 3.60 & 3 & 0 \\
4 & 0 & 4 & 0 & & \\
5 & 0 & 5 & 17.85 & & \\
6 & 0 & 6 & 0 & & \\
7 & 0 & & & & \\
8 & 3.63 & & & & \\
9 & 7.82 & & & & \\
10 & 0 & & &
\end{tabular}

*Average values for each liver are calculated as the ratio of number of incorrect predictions to the total number of times the liver was picked in 1000 runs.

The multivariate analyses performed demonstrated that ischemic and fresh rat livers are easily distinguishable from each other and accurately classifiable based on their metabolic function after exposure to different durations of ischemia. Our MPLSDA-based classification algorithm was able to determine the quality of a new liver as fresh or ischemic with $\sim 90 \%$ specificity. Once the liver quality was determined, MPCA-based SPE statistic enabled its comparison to reference fresh or reference ischemic livers, gauging the extent of recovery to a transplantable state.

We therefore conclude that the SPE statistic can be employed as an accurate and continuous index of fitness for transplantation for perfused rat livers. Based on our data, cutoff values for SPE for fresh and ischemic livers were determined to be used as heuristic limits of fitness for transplantation. This analysis can be further expanded to explore the correlations between metabolic function and traditional tests of cellular injury, and translated to human livers for a clinically applicable graft quality assessment and an index of transplantation.

The analyses performed in this work all confirm that a metabolic index of ischemic injury is a feasible idea for evaluation of perfused ischemic livers, and such a measure would be of significant use in utilization of DCD livers for transplantation. This study demonstrates the power of SPM methodologies in achieving this goal; however, further work is needed to enrich the data used here for more sophisticated metabolic analyses which may reveal underlying details of cellular injury in the context of transplantability, as well as translation to clinical studies.

\section{Abbreviations}

DBD: Donors after brain death; DCD: Donors after cardiac death; IVC: Inferior vena cava; MPCA: Multi-way principal component analysis; MPLS: Multi-way partial least squares; MPLSDA: Multi-way partial least squares discriminant 
analysis; NIPALS: Nonlinear iterative partial least squares; NMP: Normothermic machine perfusion; PC: Principal component; PCA: Principal components analysis; PLS: Partial least squares; PV: Portal vein; SPE: Squared Prediction Error; SPM: Statistical Process Monitoring; UDCD: Uncontrolled DCD.

\section{Competing interests}

Pending patent application: "Methods and compositions for preserving tissues and organs," Worldwide provisional patent application, WO/2011/ 35223 .

\section{Authors' contributions}

KU had full access to all of the data in the study and takes responsibility for the integrity of the data and the accuracy of the data analyses. Study concept and design, SP, MLI, BEU, KU Acquisition of data, MLI, HT, BEU. Analysis and interpretation of data, SP, MLI, KU Transplantation studies, HT Drafting of the manuscript, SP, KU Critical revision of the manuscript for important intellectual content, SP, MLI, BEU, MY, KU Statistical analysis, SP, KU Obtained funding, MY, KU Administrative, technical, or material support, MY and KU. All authors contributed to the preparation of the report. All authors read and approved the final manuscript.

\section{Acknowledgments}

Funding from the National Institutes of Health (R01DK096075, R01EB008678, R00DK080942, K99DK088962), and the Shriners Hospitals for Children are gratefully acknowledged.

\section{Author details}

${ }^{1}$ Center for Engineering in Medicine, Massachusetts General Hospital, Harvard Medical School, and the Shriners Hospitals for Children, 51 Blossom Street, Boston, MA 02114, USA. ${ }^{2}$ Division of Cardiac and Vascular Surgery, University Hospital Zurich, Zurich, Switzerland. ${ }^{3}$ Department of Biomedical Engineering, Rutgers University, Piscataway, NJ, USA.

Received: 10 November 2011 Accepted: 28 May 2012

Published: 25 June 2012

\section{References}

1. Abt PL, Desai NM, Crawford MD, Forman LM, Markmann JW, Olthoff KM, Markmann JF: Survival following liver transplantation from non-heartbeating donors. Ann Surg 2004, 239(1):87-92.

2. Abt PL, Fisher CA, Singhal AK: Donation after cardiac death in the US: History and use. J Am Coll Surg 2006, 203(2):208-225.

3. Tolboom H, Izamis ML, Sharma N, Milwid JM, Uygun B, Berthiaume F, Uygun K, Yarmush ML: Subnormothermic Machine Perfusion at Both 20 degrees $C$ and 30 degrees $C$ Recovers Ischemic Rat Livers for Successful Transplantation. J Surg Res 2012, 175(1):149-156. Epub 2011 Mar 29.

4. Mateo R, Cho Y, Singh G, Stapfer M, Donovan J, Kahn J, Fong TL, Sher L, Jabbour N, Aswad S, et al: Risk Factors for Graft Survival After Liver Transplantation from Donation After Cardiac Death Donors: An Analysis of OPTN/UNOS Data. Am J Transplant 2006, 6(4):791-796.

5. Bessems M, Doorschodt BM, van Vliet AK, van Gulik TM: Improved rat liver preservation by hypothermic continuous machine perfusion using Polysol, a new, enriched preservation solution. Liver Transp/ 2005, 11(5):539-546.

6. Bessems M, Doorschodt BM, Kolkert JLP, Vetelainen RL, van Vliet AK, Vreeling H, van Marle J, van Gulik TM: Preservation of steatotic livers: A comparison between cold storage and machine perfusion preservation. Liver Transp/ 2007, 13(4):497-504.

7. Dutkowski P, Graf R, Clavien PA: Rescue of the cold preserved rat liver by hypothermic oxygenated machine perfusion. Am J Transplant 2006, 6(5 Pt 1):903-912.

8. Dutkowski P, Furrer K, Tian Y, Graf R, Clavien PA: Novel Short-term Hypothermic Oxygenated Perfusion (HOPE) System Prevents Injury in Rat Liver Graft From Non-Heart Beating Donor. Ann Surg 2006, 244(6):968-977.

9. de Rougemont O, Breitenstein S, Leskosek B, Weber A, Graf R, Clavien PA, Dutkowski P: One hour hypothermic oxygenated perfusion (HOPE) protects nonviable liver allografts donated after cardiac death. Ann Surg 2009, 250(5):674-683.

10. Lee CY, Jain S, Duncan HM, Zhang JX, Jones JW, Southard JH, Clemens MG: Survival transplantation of preserved non-heartbeating donor rat livers: Preservation by hypothermic machine perfusion. Transplantation 2003, 76(10):1432-1436.
11. Xu H, Lee CY, Clemens MG, Zhang JX: Pronlonged hypothermic machine perfusion preserves hepatocellular function but potentiates endothelial cell dysfunction in rat livers. Transplantation 2004, 77(11):1676-1682.

12. Tolboom H, Pouw R, Izamis M, Milwid J, Sharma N, Soto-Gutie'rrez A, Nahmias Y, Uygun K, Berthiaume F, Yarmush ML: Recovery of Warm Ischemic Rat Liver Grafts by Normothermic Extracorporeal Perfusion. Transplantation 2009, 87(2):170-177.

13. Tolboom H, Milwid JM, Izamis ML, Uygun K, Berthiaume F, Yarmush ML: Sequential cold storage and normothermic perfusion of the ischemic rat liver. Transplant Proc 2008, 40(5):1306-1309.

14. Tolboom H, Pouw R, Uygun K, Tanimura Y, Izamis ML, Berthiaume F, Yarmush ML: A model for normothermic preservation of the rat liver. Tissue Eng 2007, 13(8):2143-2151.

15. Fondevila C, Hessheimer AJ, Maathuis MHJ, Munoz J, Taura P, Calatayud D, Leuvenink H, Rimola A, Ploeg RJ, Garcia-Valdecasas JC: Superior preservation of DCD livers with continuous normothermic perfusion. Ann Surg 2011, 254(6):1000-1007.

16. Guarrera JV, Henry SD, Samstein B, Odeh-Ramadan R, Kinkhabwala M, Goldstein MJ, Ratner LE, Renz JF, Lee HT, Brown RSJ, et al: Hypothermic machine preservation in human liver transplantation: the first clinical series. Am J Transplant 2009, 10(2):372-381.

17. Imber CJ, St Peter SD, Lopez de Cenarruzabeitia I, Pigott D, James T, Taylor R, McGuire J, Hughes D, Butler A, Rees M, et al: Advantages of normothermic perfusion over cold storage in liver preservation. Transplantation 2002, 73(5):701-709.

18. Pienaar BH, Lindell SL, Van Gulik T, Southard JH, Belzer FO: Seventy-twohour preservation of the canine liver by machine perfusion. Transplantation 1990, 49(2):258-260.

19. Eriksson L, Johansson E, Kettaneh-Wold N, Trygg J, Wikstrom C, Wold S: Multi- and Megavariate Data Analysis Part I. Basic Principles and Applications. Umea, Sweden: Umetrics Academy; 2006.

20. Cinar A, Parulekar SJ, Undey C, Birol G: Batch Fermentation: Modeling, monitoring, and control. New York, NY: Marcel Dekker; 2003.

21. Heijnen BHvVS, Straatsburg IH, van Gulik TM: Pronounced effect of minor changes in body temperature on ischemia and reperfusion injury in rat liver. J Appl Physiol 2001, 91(1):265-268.

22. Delriviere L, Gibbs P, Kobayashi E, Goto S, Kamada N, Gianello P: Detailed modified technique for safer harvesting and preparation of liver graft in the rat. Microsurgery 1996, 17(12):690-696.

23. Delriviere L, Gibbs P, Kobayashi E, Goto S, Kamada N, Gianello P: Technical details for safer venous and biliary anastomoses for liver transplantation in the rat. Microsurgery 1998, 18(1):12-18.

24. Kamada N, Calne RY: Orthotopic liver transplantation in the rat. Technique using cuff for portal vein anastomosis and biliary drainage. Transplantation 1979, 28(1):47-50.

25. Nomikos P, MacGregor JF: Multivariate SPC Charts For Monitoring Batch Processes. Technometrics 1995, 37:41-59.

26. Nomikos $P$, MacGregor JF: Multi-way partial least squares in monitoring batch processes. Chemometr Intell Lab 1995, 30:97-108.

27. Wold S, Geladi P, Esbensen K, Ohman J: Multi-way principal components and PLS analysis. J Chemometr 1987, 1:41-56.

28. Efron B: The jackknife, the bootstrap, and other resampling plans, Volume 38. Philadelphia: SIAM; 1982.

29. Efron B, Tibshirani RJ: An introduction to the bootstrap. Boca Raton, FL: Chapman \& Hall/CRC Press; 1993.

30. Jackson JE: Principal components and factor analysis: Part I - Principal Components. J Quality Technol 1980, 12(4):201-213.

31. Nomikos P, MacGregor JF: Monitoring Batch Processes Using Multi-way Principal Component Analysis. AICHE J 1994, 40:1361-1375.

32. Box GEP: Some theorems on quadratic forms applied in the study of analysis of variance problems: Effect of inequality of variance in one-way classification. Ann Math Statist 1954, 25:290-302.

\section{doi:10.1186/1756-0500-5-325}

Cite this article as: Perk et al: A fitness index for transplantation of machine-perfused cadaveric rat livers. BMC Research Notes 2012 5:325. 